\title{
Double Polarization SAR Image Classification Based on Object-Oriented Technology
}

\author{
Xiuguo Liu ${ }^{1,2}$, Yongsheng $\mathrm{Li}^{2}$, Wei Gao ${ }^{2}$, Lin Xiao ${ }^{2}$ \\ ${ }^{1}$ Faculty of Information Engineering, China University of Geosciences, Wuhan, China \\ ${ }^{2}$ Wuhan Zondy Cyber T\&S. Co., Ltd, Wuhan, China \\ E-mail: liuxg318@163.com
}

\begin{abstract}
This paper proposed to use double polarization synthetic aperture radar (SAR) image to classify surface feature, based on DEM. It takes fully use of the polarization information and external information. This paper utilizes ENVISAT ASAR APP double-polarization data of Poyang lake area in Jiangxi Province. Compared with traditional pixel-based classification, this paper fully uses object features (color, shape, hierarchy) and accessorial DEM information. The classification accuracy improves from the original $73.7 \%$ to $91.84 \%$. The result shows that object-oriented classification technology is suitable for double polarization SAR's high precision classification.
\end{abstract}

Keywords: Synthetic Aperture Radar, Image Classification, Object-Oriented, Pixel-Based, DEM

\section{Introduction}

SAR has great significance because of its imaging capability in all day and all weather which could make up for the weakness of optical remote sensing in the application of land use and dynamic monitoring in cloudy and rainy areas. Electromagnetic waves are sensitive to the shape, size, texture, surface roughness, the complex permittivity of ground objects. Compared with single-Polarization, dual polarization is more sensitive to the properties of the ground objects and it's more suitable for ground objects' recognition and land use classification.

Traditional information extraction technology with single a pixel as a unit emphasizes too much on part (color and texture of a single pixel), while ignores the geometric structure of whole map spot nearby.

Pixel-oriented solution model analyze pixel separately which has low interpretation accuracy.

Pixel-based classification methods, such as maximum likelihood and ISODATA, only use the backscatter coefficient of SAR images, and the rich texture, roughness and other information in images are not applied. The result of classification is not satisfactory. The smallest processing unit of object-oriented classification method in information extraction is no longer the pixel, but the object with more semantic information of adjacent pixels. It classifies the remote sensing images in a higher level in order to reduce the semantic information loss rate as in the traditional pixel-based classification; so that the clas- sification results semantic information will be richer [1].

A lot of scholars from home and abroad have studied the land use and land-cover classification using optical images based on object-oriented technology. The results show the accuracy of classification improves significantly compared with the traditional classification methods [2,3]. There are also many scholars who have studied the objectoriented classification using SAR images. [4,5]. However, the theoretical foundation, model building and other aspects in this field are less advanced than optical images obviously. This paper tries to conduct the double-polarized SAR image classification based on object-oriented technology, and confirms that the method is also suitable for the high-precision classification of dual-polarized SAR images.

\section{Object-Oriented}

The object-oriented classification idea is to simulate human cognitive processes. The human brains always put things in a specific environment when analyzing and identifying the things. Environment is vital important for identifying things. Human's perception of external things is unified, including the color, external contour, spacing and other properties of things. Similarly, in making visual interpretation of remote sensing images, besides the differences between the colors, it can identify the ground objects by texture, shape, adjacency relationships etc. The object-oriented classification method extracts the homo- 
geneous regions before classifying. The specific two-step process is multi-scale segmentation and classification.

\subsection{Multi-Scale Segmentation of Image}

The multi-scale Segmentation of SAR image aims to cut the image into multiple small areas. Each small area has the same attributes and these regions can be taken as the ground objects in reality. Image segmentation shows that each pixel is an object at the beginning. The process is to combine the similar and nearby objects into a large new object. Compared with the pixels, image object has multicharacters, such as color, size, shape, uniformity and so on. Generally, SAR images have macroscopic and microscopic characteristics. If cut the region with singlescale, it will result in lots of broken areas. It is not conducive to information interpretation. The information of different ground objects need to be analyzed in different space sub-scales.

In the multi-scale segmentation process, each object layer has a scale. The remote sensing images can be described by a variety of the same phase and appropriate scales that caused by several object layers, rather than a single scale. The larger the segmentation scale, the larger the region area in the generated object layers and vice versa. It will form a multi-scale layers diagram of objects after several times' segmentation. The diagram is showed as Figure 1.

By using multi-scale segmentation technique, form image objects at different scales, at the same time generate the adjacent relation and level inheritance relation which have impacts on the objects. Different scales of image segmentation reduce data unit number to be addressed in the classification, and speed up the classification.

Choose appropriate level to extract information between multiple layers scales after the multi-scale segmentation. The larger spatial scale surface features can be extracted by the large split-scale layer, such as rivers, forests, etc. Similarly, proposing to select the smaller-scale layers for small split-scale or areas with complex feature.

\subsection{Object-Oriented Classification}

Object-oriented classification have two mainly methods, including nearest neighbor distance method and member

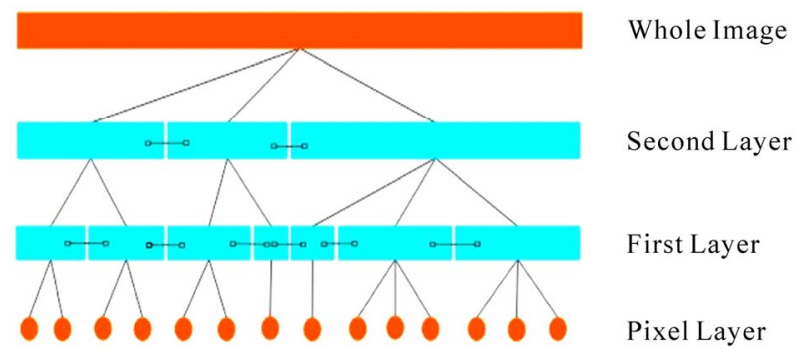

Figure 1. Hierarchy network graph of image object. function method. The nearest distance classification is similar to supervised classification, which needs to select the sample. The member function method is based on the object characteristics with fuzzy logic, which is an uncertain method of the analysis of things. The polygon objects after being split do not rigidly belong to a certain ground object type, but describing the similar degree of object types and a certain ground object. Fuzzy logic is a kind of mathematical methods to quantify uncertainty. Using the member function method requires only a small amount of feature information to identify the types of ground objects. The member function method is a regulate classification method by imaging the object spectrum, shape, texture features and other information.

At the same time, the object-oriented classification can also use other ancillary information, such as DEM, statistical information of ground object area, as well as the known maps of ground object classification. Using DEM can separate mountains, hills, and plains based on the terrain trend. Using the statistical information of ground object area can make supplementary classification according to the area.

\section{Experiment Result}

\subsection{Experiment Area Survey}

This paper selects a nearby typical area from the Poyang Lake region, Jiangxi Yongxiu County as an experiment area. This region is located in northern part of Jiangxi Province, Poyang Lake, west (longitude $115^{\circ} 45^{\prime}-115^{\circ} 55^{\prime}$, north latitude $28^{\circ} 59^{\prime}-29^{\circ} 10^{\prime}$ ). It is subtropical monsoon climate zone, four distinctive seasons and sufficient sunlight, abundant rainfall, and is very suitable for the development of grain, cotton, oil and aquaculture. Land-use types mainly include hills, rivers, towns, grasslands, farmlands, as well as floodplain and other unused land. (as shown in Figure 2(a))

\subsection{Data Processing}

Choose SAR data in the Poyang Lake region, Jiangxi Yongxiu County ENVISAT APP dual-polarized data which acquired on July 28, 2004 as the experimental data in this paper. Its polarization is $\mathrm{HH}, \mathrm{VV}$ and resolution is 12.5 meters (Figure 2(b)). Before the classification of SAR images, it needs to do pre-processing, including image calibration, geometric correction and filtering. In order to increase the amount of information in dual-polarized images, it increases a band $\mathrm{HH}-\mathrm{VV}$ based on the original two polarizations. The results showed that the differences between surface features are more clearly.

At the same time, this paper uses DEM as an auxiliary parameter to extract the hilly regions. DEM layer is set 


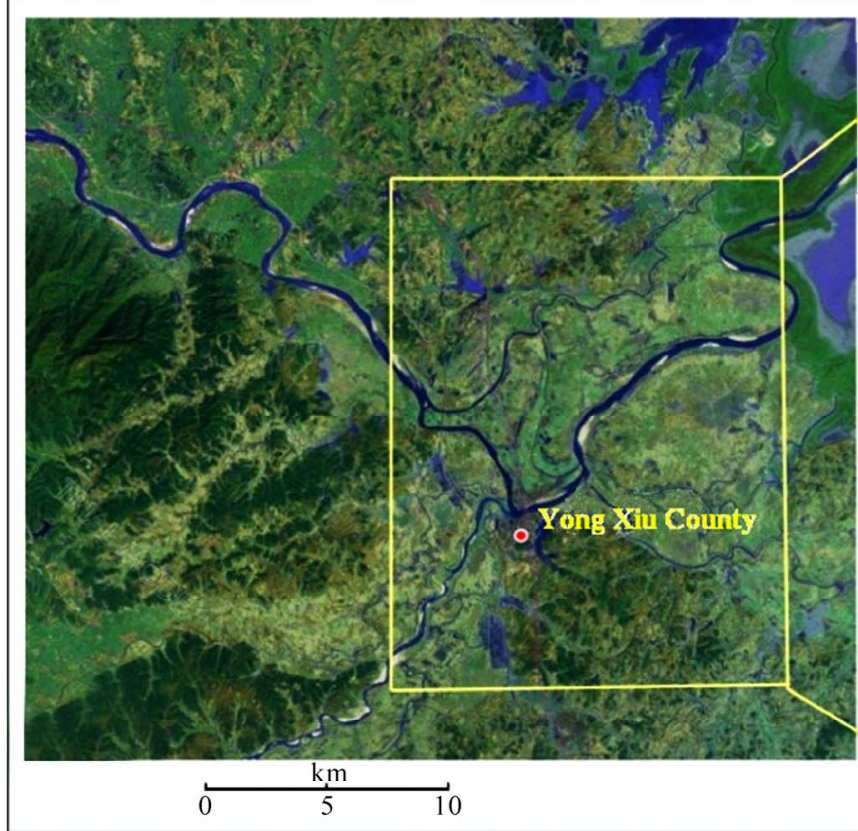

(a)

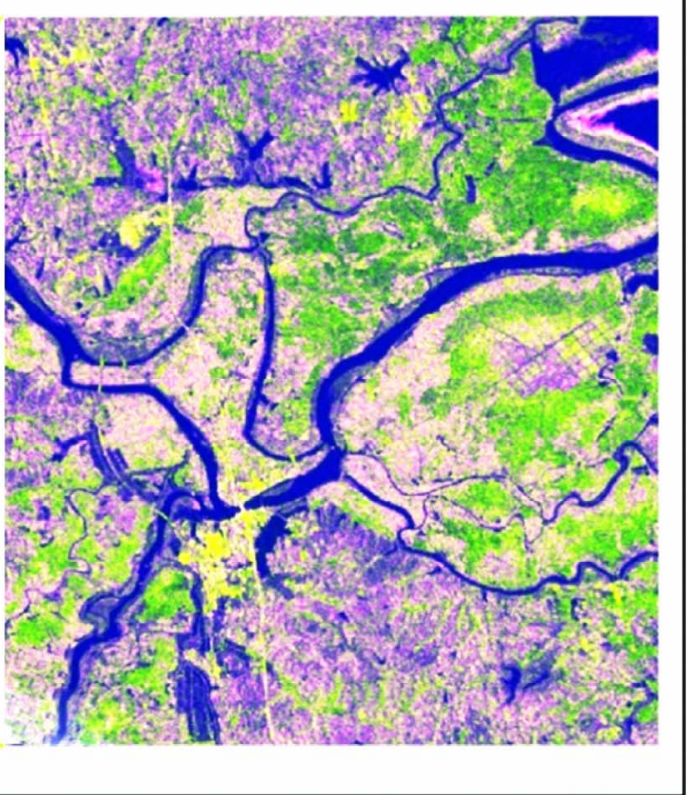

(b)

Figure 2. The local of experiment area (a) optical image, (b) ENVISAT false colour synthetical image red: HH Green: VV Blue: HH-VV.

as Layer 4 and the elevation of hills in the region is above 50 meters. It is shown in Figure 3.

\subsection{The Traditional Classification Method}

The traditional method is based on the pixel. It only takes advantage of the pixel values of the backward scattering. Specific classification methods include unsupervised classification and supervised classification. The traditional method completes classification based on ZONDY_SAR radar

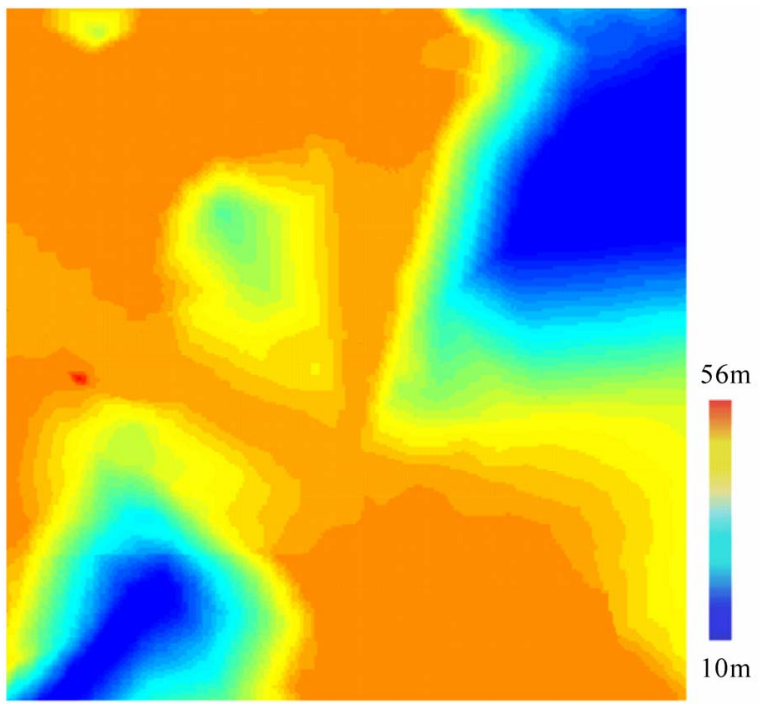

Figure 3. Auxiliary DEM image. remote sensing image processing platform.

Non-supervised classification conduct "blind" classification under the condition that people do not have any prior knowledge before the classification process, according to the statistical characteristics and natural clustering features of the images. Its classification is only distinguishing different categories, but does not determine the types of property. Supervised classification is the method of pattern recognition, by selecting the characteristic parameters to find the characteristic parameters as the decision-making rules before the choice of representative or typical features in the training area and establishing discriminate function classification of individual images.

Non-supervised classification adopts ISODATA method. As shown in Figure 4. Unsupervised classification only recognizes 4 features, while the hills and bare land have not been correctly identified. Supervised classification sampling method is BP neural network. As shown in Figure 5. Supervised classification can identify most of the surface features, but the results have a serious error, that is hills are assigned to farmland category.

Because the overall scattering characteristics in the image of the fields and hills are similar, the supervised classification methods cannot separate the two kinds of surface features. The external solution is to use DEM, which will be specifically introduced in the following description. At the same time, supervised classification and unsupervised classification has serious "salt and pepper" noise to some extent, which is the inherent limitation of traditional classification methods. 


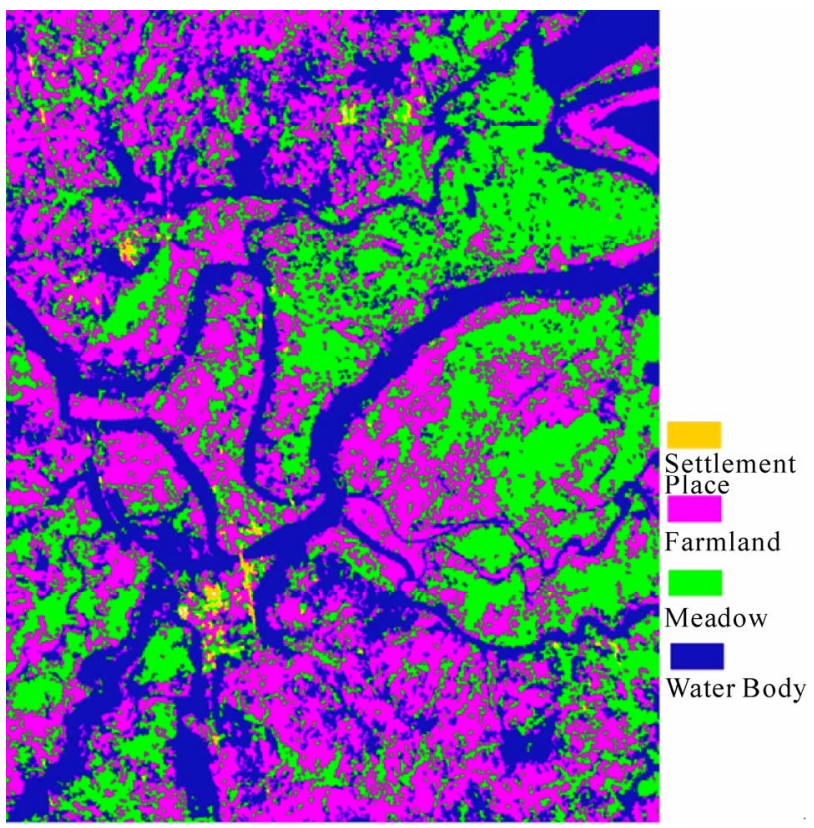

Figure 4. Unsupervised classification result (ISODATA).

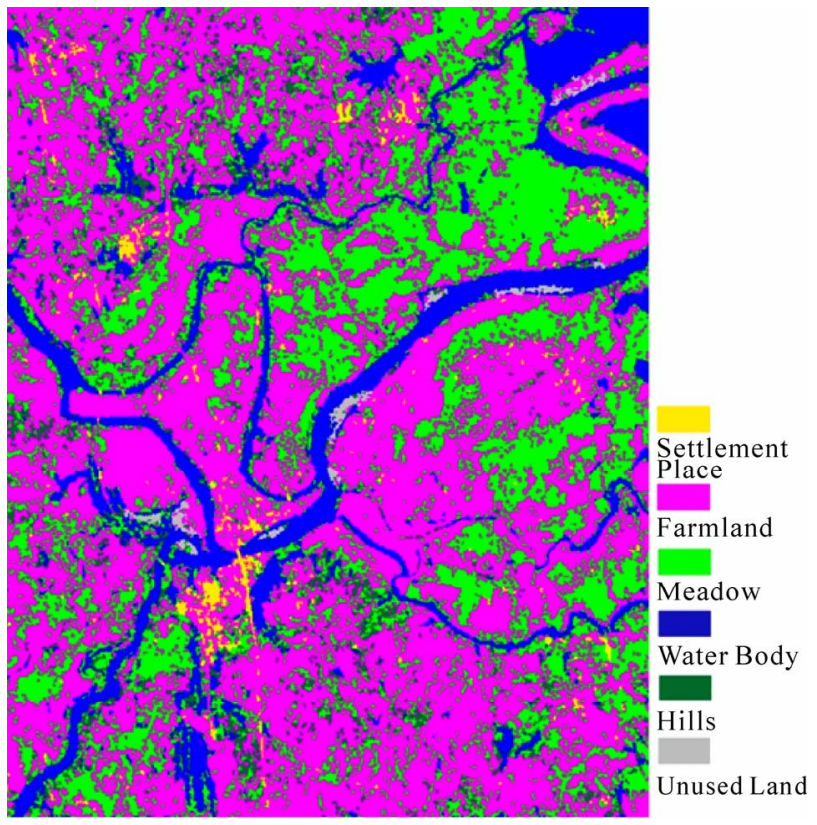

Figure 5. Supervised classification result (BP neural network).

\subsection{Object-Oriented Classification}

The basic entity of object-oriented classification of is a meaningful image objects, rather than single pixel. Image object not only contains scattering information, but also contextual information, object texture information and some auxiliary information. The following experiment is completed based on the Definiens 7.0 object-oriented information extraction system software.

\subsubsection{Multi-Scale Segmentation}

The scales of multi-scale segmentation of images are 30 and 60. There is a big difference between objects that obtained by different scales. There are a larger number of objects in images when the scale is 30 (Figure 6(a)). Small area such as residents can be separated from surface features such as energy and the surrounding grasslands, farmlands, etc, while large-scale surface features such as water bodies in the segmentation results have been divided into a number of small objects. It is not conducive to water extraction.

Large area water can be extracted when the scale is 60 (Figure 6(b)). It can be expressed with a small amount of polygonal objects and the effect is good. But small object like residents is merged into the category of grass which results in a mixed object. This is not beneficial to the extraction of the residents. Therefore, these features need to use smaller-scale segmentation.

\subsubsection{Classification}

According to the characteristics of experiment area features and the relationship between information and objects, this paper uses hierarchical classification structure. It is built on two levels. The divided scale of the first layer Level1 is 30. It is used for the unused land such as residents and exposed areas. The divided scale of the second layer Level2 is 60 , and is used for the hills, water, farmland, grasslands and other regions. The specific processes are shown in Figure 7.

The first is the extraction of water. The water color in the image is a little bit dark. The water body is extracted by setting the rules. Due to the fact that there are a small number of residents in some parts of hills, residents are extracted first. Then hills in the remaining land are extracted second. This step needs the help of an external DEM. Lastly, extract residential areas, grasslands and farmlands in the remaining plain areas. Specific rules are as follows,

Water: Mean Layer $1<-17$

Residents: Standard deviation Layer1 $>=41.5$

Hill: Mean Layer4 > 49.5 (DEM)

Farmland: Mean of Inner border Layer2 $>170$

Grassland: Ratio Layer 1> $=0.57$.

After the above steps, most of the features have been extracted. The features without division should be categorized into unused land. Through the cell processing and cartographic generalization, the classification results are shown in Figure 8.

As can be seen from Figure 8, the object-oriented classification eliminates the "salt and pepper" noise which exists in the traditional classification and smooth the results in detail. The category of features is also more abundant. With the help of DEM, object-oriented classification can accurately extract the hills, which cannot be achieved by traditional classification methods. 


\subsection{Accuracy Evaluation}

Choose the results of supervised classification and objectoriented classification to do accuracy evaluation comparison. The two methods are both based on samples.

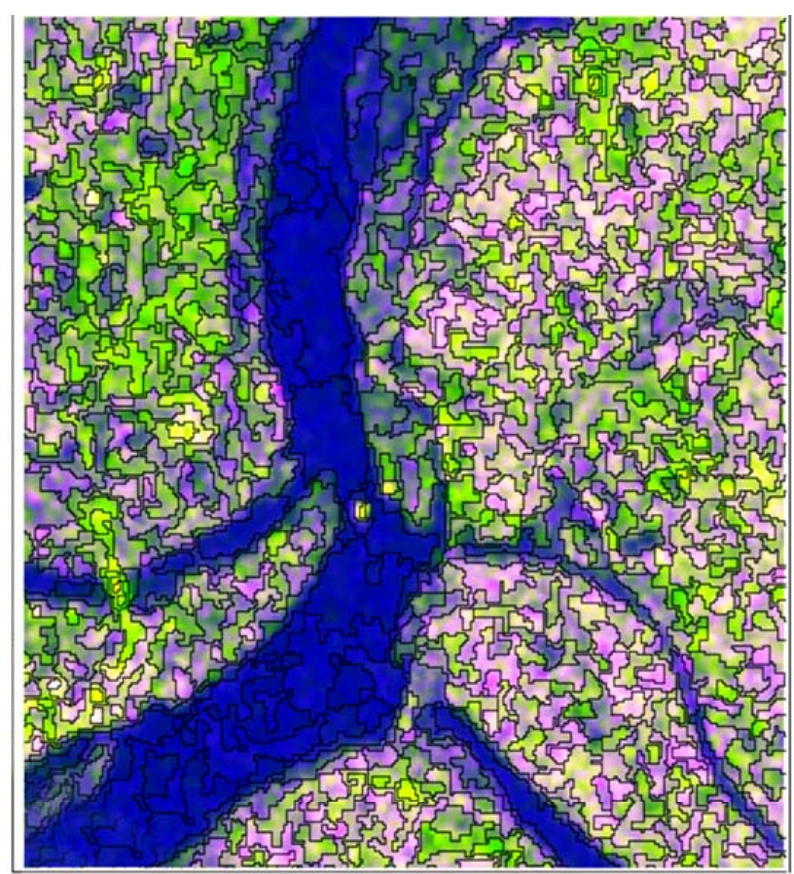

(a)

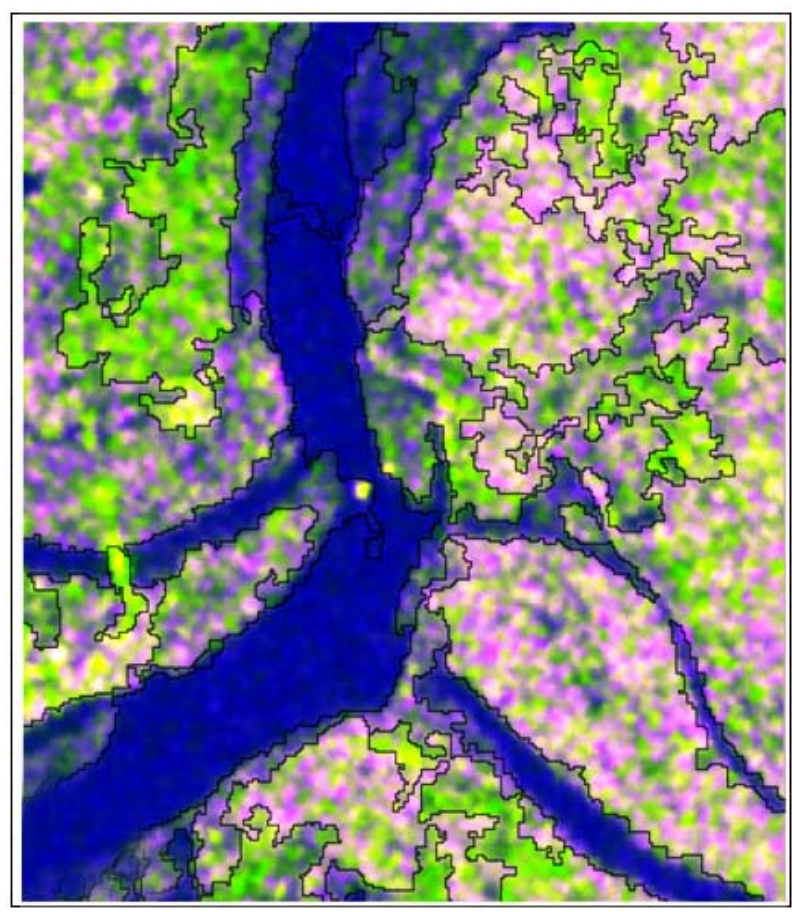

(b)

Figure 6. Result of Different-Scale Segmentation. (a) scale 30; (b) scale 60.

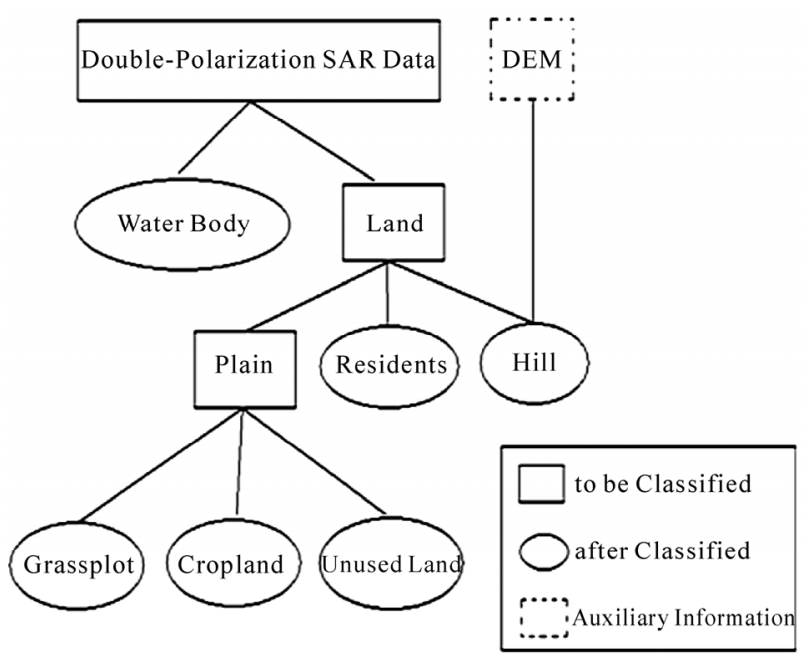

Figure 7. Processing flow chart of object-oriented classification.

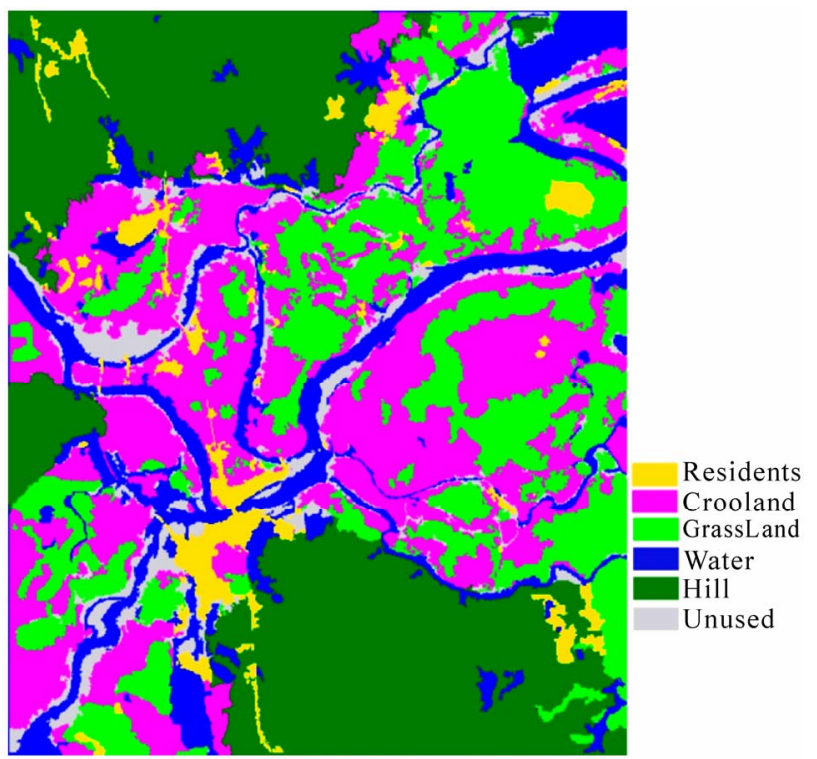

Figure 8. Result of object-oriented classification.

Supervised classification is based on a sample of pixel, while when it comes to object-oriented methods; the sample is based on image objects shown in Table $\mathbf{1}$ and Table 2 .

As shown in the Table 1 and Table 2 which record the classification accuracy of evaluation results, the overall classification accuracy and Kappa coefficient of the traditional supervised classification is $73.7 \%$ and 0.654 . While the overall object-oriented classification accuracy and Kappa coefficient is $91.84 \%$ and 0.895 , respectively. This shows that the object-oriented technology improves the accuracy of SAR image classification significantly. The traditional method is very easy to mislead hill to farmland because of the similar scattering characteristics. By using the external DEM, the object-oriented approach 
Table 1. Precision of evaluate supervised classification.

\begin{tabular}{lcccccccc}
\hline & Water & Residents & Hill & GrassPlot & Cropland & Unused land & Samples sum & User precision \% \\
\hline Water & 6465 & 0 & 11 & 0 & 0 & 550 & 7026 & 92.02 \\
Residents & 0 & 1243 & 0 & 54 & 0 & 0 & 1297 & 95.84 \\
Hill & 0 & 0 & 1375 & 0 & 0 & 0 & 1375 & 100.00 \\
Grassplot & 0 & 0 & 922 & 14989 & 0 & 251 & 15911 & 94.19 \\
Cropland & 0 & 119 & 7286 & 1614 & 5389 & 0 & 14659 & 36.76 \\
Unused land & 37 & 0 & 0 & 0 & 22 & 935 & 994 & 94.16 \\
Samples sum & 6502 & 1362 & 9594 & 16657 & 5411 & 1736 & & 41262 \\
procretor \% & 99.43 & 91.26 & 14.33 & 89.99 & 99.59 & 53.86 & &
\end{tabular}

Table 2. Precision of object-oriented classification.

\begin{tabular}{|c|c|c|c|c|c|c|c|c|}
\hline & Water & Residents & Hill & Grassland & Cropland & Unused land & Samples sum & User precision \% \\
\hline Water & 81 & 0 & 0 & 0 & 0 & 2 & 83 & 97.59 \\
\hline Residents & 0 & 25 & 1 & 4 & 0 & 0 & 30 & 83.33 \\
\hline Hill & 0 & 1 & 17 & 0 & 2 & 0 & 20 & 85.00 \\
\hline Grassland & 0 & 1 & 1 & 59 & 3 & 0 & 64 & 92.29 \\
\hline Cropland & 0 & 0 & 0 & 3 & 28 & 1 & 15 & 87.50 \\
\hline Unused land & 0 & 0 & 0 & 0 & 1 & 15 & 16 & 93.75 \\
\hline Samples sum & 81 & 27 & 19 & 66 & 34 & 18 & 245 & \\
\hline procretor \% & 100 & 92.59 & 89.47 & 89.39 & 82.35 & 83.33 & & \\
\hline \multicolumn{2}{|c|}{ Precision sum $=91.84 \%$} & kappa $=0.895$ & & & & & & \\
\hline
\end{tabular}

separate farmland and hills through the elevation information. This increases the classification accuracy of the hills greatly, and this method cannot be achieved by traditional classification methods. As is shown from the accuracy of evaluation results, the producer accuracy of the hills in supervised classification is only $14.33 \%$. The accuracy of supervised classification results is not high mainly due to the inaccuracy of this indicator.

Although the classification accuracy of some indicators of the object-oriented technology is lower than the supervised classification, this does not affect the overall classification accuracy.

\section{Result and Argumentation}

This paper uses external DEM, and classifies dual-polarization SAR images by using object-oriented technology. The classification accuracy is $91.84 \%$, while the traditional pixel-based classification accuracy is only $73.7 \%$.
1) The traditional classification can only make use of image pixel gray values, rather than other ancillary information, such as external DEM elevation in this paper, except its own image pixel gray value. The object-oriented classification not only takes the multiple features into account, but also can judge by making use of external auxiliary information. The results showed that object-oriented classification method can estimate the type of ground object effectively.

2) The results of object-oriented classification eliminate the inherent "salt and pepper" noise of pixel-based classification. What's more, the classification of object-oriented technology not only make use of the scattering information of image, but also takes advantage of the shape, texture information, and external auxiliary information of the object. The experimental results adequately depict that the object-oriented technology is also suitable for high-precision SAR image classification.

With the development of SAR technology, full-polarization and high-resolution SAR images can provide more 
detailed features. Future research direction is applying object-oriented technology in the full-polarization SAR or high-resolution SAR image classification.

\section{Acknowledgements}

Thanks for providing experimental data by Envisat satellite radar data-sharing project of Center for Earth Observation and Digital Earth Chinese Academy of Sciences to this paper.

\section{References}

[1] L. P. You, "Object Oriented Classification Method from High Resolution Remote Sensing Imagery,” Master Thesis, Fujian Normal University, Fuzhou, 2007.

[2] C. B. Ursula, H. Peter, W. Gregor, et al., "Multi-Resolution, Object-Oriented Fuzzy Analysis of Remote Sensing Data for GIS-Ready Information,” ISPRS Journal of Photogrammetry \& Remote Sensing, Vol. 58, No. 3-4, 2004, pp. 239-258.

[3] G. Z. Shen and J. J. Liao, “An Object Oriented Methodology for Automatic Analysis of Inundate Extent Using Multi-Polarized SAR Image," Remote Sensing Technology and Application, Vol. 22, No. 1, 2007, pp. 79-82.

[4] T. Michael, E. Thomas and D. Stefan, "Object-Oriented Detection of Settlement Areas from TerraSAR-X DATA, Initial Reports," Remote Sensing-New Challenges of High Resolution, 2008, pp. 242-248.
[5] F. L. Chen, C. Wang and H. Zhang, "The Analysis of Single Polarization Synthetic Aperture Radar Images for the Application of Land-Use and Land-Cover Change," Remote Sensing Technology and Application, Vol. 23, No. 3, 2008, pp. 289-293.

[6] J. Qian, Q. M. Zhou and Q. Hou, "Comparison of PixelBased and Object-Oriented Classification Methods for Extracting Built-up Areas IN AridZone,” ISPRS Workshop on Updating Geo-Spatial Databases with Imagery \& The $5^{\text {th }}$ ISPRS Workshop on DMGISs, 2007, pp. 163-171.

[7] L. Stanistaw, "Object-Oriented Classification of Landsat ETM+ Satellite Image,” Journal of Water and Land Development, Vol. 20, No. 10, 2006, pp. 91-106.

[8] Q. L. Tan, Z. G. Liu and W. Shen, "An Algorithm for Object-Oriented Multi-Scale Remote Sensing Image Segmentation,” Journal of Beijing Jiaotong University, Vol. 31, No. 4, 2007, pp. 111-114.

[9] F. L. Du, Q. G. Tian and X. Q. Xia, “Object-Oriented Image Classification Analysis and Evaluation,” Remote Sensing Technology and Application, Vol. 19, No. 1, 2004, pp. 20-23.

[10] C. Y. Zhou, “Object Oriented Information Extraction Technology from High Resolution Remote Sensing Imagery,” Master Thesis, Shan Dong University of Science and Technology, Tsingtao, 2006.

[11] H. M. Zhang, Z. Z. Bian, et al., "A Novel Multi-Resolution Fuzzy Segmentation Method on MR Image,” Journal of Computer Science and Technology, Vol. 18, No. 5, 2003, pp. 659-666. 